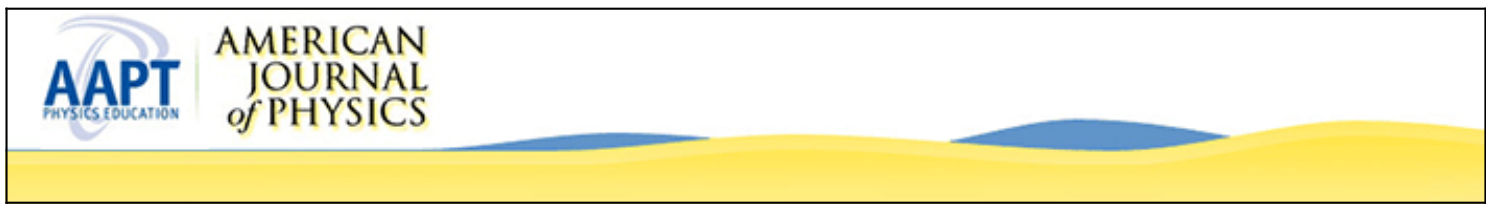

\title{
Construction of Thermodynamic Models for Elementary Teaching
}

Jesse W. M. DuMond

Citation: American Journal of Physics 9, 234 (1941); doi: 10.1119/1.1991687

View online: http://dx.doi.org/10.1119/1.1991687

View Table of Contents: http://scitation.aip.org/content/aapt/journal/ajp/9/4?ver=pdfcov

Published by the American Association of Physics Teachers

\section{Articles you may be interested in}

An interactive method for teaching circuit model construction for complex interconnected acoustic systems J. Acoust. Soc. Am. 131, 3293 (2012); 10.1121/1.4708312

The Elementary Teaching of String Theory

AIP Conf. Proc. 1263, 242 (2010); 10.1063/1.3479879

A model for the construction of elementary concepts

AIP Conf. Proc. 465, 45 (1999); 10.1063/1.58258

An elementary construction of the color wave functions in the quark model

Am. J. Phys. 64, 589 (1996); 10.1119/1.18459

Teaching physics in the elementary grades

Phys. Today 17, 34 (1964); 10.1063/1.3051172

\section{WebAssign.}

\section{Free Physics Videos}

Add these videos and many more resources - free with WebAssign. 


\title{
Construction of Thermodynamic Models for Elementary Teaching
}

\author{
Jesse W. M. DuMond \\ California Institute of Technology, Pasadena, California
}

$\mathrm{T}$ HE author has found great value in the use of solid models in freshman physics teaching to illustrate (1) the law of ideal gases, and (2) the van der Waals equation of state and the behaviors of vapors and liquids. Many freshman students have difficulty in visualizing the shape of the thermodynamic surfaces from, let us say, a blackboard sketch of a set of isotherms, and the better part of a precious lecture hour may be consumed in explaining, with many words, what would be almost obvious if one had a solid model in his hands. The author has found that more than half of a class of average intelligence, having learned that an ideal gas is described by the equation $P V=N R T$, will be sorely puzzled by the adiabatic equation for ideal gases, $P V^{\gamma}=$ const. It helps these puzzled students surprisingly little to be told that, since the first equation contains three variables, it is not inconsistent with the second. With many of them, however, the matter becomes clear when they are shown an ideal gas surface on which have been plotted isotherms, adiabatics, and so forth.

The models shown in Figs. 1 to 4 were constructed by the author. It is probable that a model like the ideal gas surface of Fig. 1 can be purchased from supply houses, ${ }^{1}$ but it is not at all difficult to construct. The model of the van der Waals surface, however, contains features which the author believes to be novel.

The ideal gas surface shown in Fig. 1 is made of plaster of Paris. Three square boards form the main supporting frame. These are joined with nails and glue in the shape of the three square surfaces which meet at one corner of a cube. As can be seen, the outward corner of one surface is cut away, leaving a profile in the form of an equilateral hyperbolic arc. The dihedral angles adjacent to this arc are terminated with pieces of wood in the form of right triangles as shown. To reduce the weight of the model, it

\footnotetext{
1 See also: Verwiebe, "Models of thermodynamic surfaces," Am. J. Phys. (Am. Phys. T.) 3, 179 (1935); Zemansky and Herman, "The Gibbs and Mollier thermodynamic surfaces," Am. J. Phys. (Am. Phys. T.) 4, 194 (1936).
}

is better not to fill the box, so formed, full of plaster but rather to construct a backing of tar paper and wire netting nailed onto a few triangular wooden braces to form a surface roughly approximating the plaster surface but so situated as to give room on top of it for a coating of plaster $\frac{1}{2}$ to $\frac{3}{4}$ in. thick. Before nailing down the tar paper and wire netting, the woodwork should be painted inside and out with several coats of paint to protect it against absorbing moisture from the plaster and thus to prevent warping. On the visible outer surfaces of the wood, it is desirable to use "blackboard" paint which, when dry, gives a surface suitable for chalk marks indicating numbers, symbols, coordinates, and so forth. Two round holes not visible in Fig. 1 should be bored in the board farthest from the observer. These serve to ventilate the space behind the plaster, thus facilitating the drying, and also may be used as grip holes into which the fingers may be inserted for conveniently carrying the model.

The plaster may be mixed a little at a time and applied in several coats, without too much delay for hardening between applications. When sufficient plaster has been built up, the ideal gas surface is very easily generated by the use of a straight bar of rectangular, cold rolled brass or

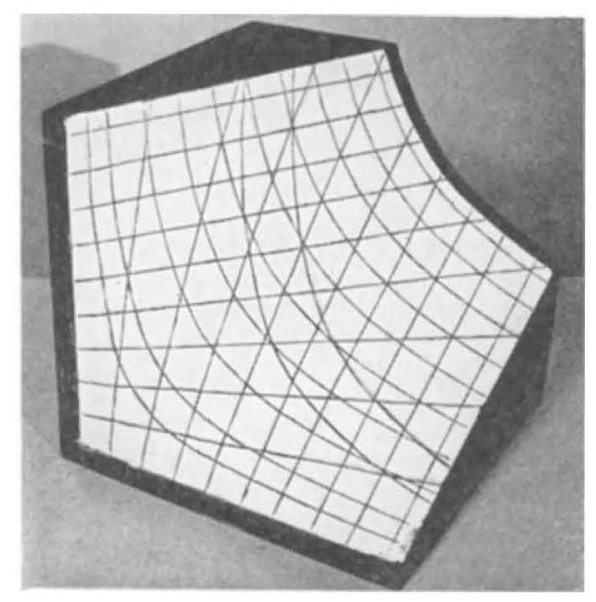

FIG. 1. The ideal gas surface. 
steel. This bar is held parallel, let us say, to the surfaces of constant pressure and caused to move so that near one end it slides up and down the vertical (pressure) axis, while near the other end it follows first the hyperbolic arc and then the hypotenuse of the right triangle. The bar in so doing successively assumes the positions of all the lines of constant pressure shown in the photograph. As the bar moves, it is made to cut away the excess plaster until the surface has the correct shape. It is, of course, possible also to move the bar so that it assumes the positions of successive lines of constant volume, and it is preferable to test the figure of the surface both ways before leaving it to the hardening process. A basin of water should be kept handy to wash off the excess plaster from the bar. After the plaster has hardened, the isotherms are scribed with a machinist's surface gauge. For this operation, the model should preferably lie on that one of its three flat faces from which the hyperbolic arc was cut, and this face should lie on a large plane surface upon which the surface gauge can slide. The lines of constant pressure and of constant volume, since they are straight, can readily be scribed with a straight edge, but a surface gauge may also be used conveniently for them. The grooves so scribed can then be filled in with pencil, paint or India ink applied to the plaster. Two or more adiabatic curves, as shown in Fig. 1, should also be plotted using the adiabatic equation. The coordinates defined by the straight lines of constant pressure and of constant volume are used as reference coordinates for this purpose. The adiabatics here shown were plotted for a value $\gamma=5 / 3$.

The van der Waals surface was not made of plaster because the removable blocks which form an important feature (about to be described) have, unfortunately, some rather sharp knifelike edges, which would be too fragile if plaster were used. This surface was therefore built up out of boards glued together with the grain running vertically in alternate boards and horizontally in the remainder (Fig. 2). The flat surfaces of the boards are parallel to planes of constant temperature. The boards are first cut out in the form of true square slabs and the various isotherms are traced, one on each board. The profiles of the isotherms are then sawed out with a band saw, and it is advisable to save both pieces. It is convenient for plotting to use the van der Waals equation in the "reduced" form,

$$
\left(P+3 / V^{2}\right)\left(V-\frac{1}{3}\right)=8 T / 3,
$$

in which the variables $P, V$ and $T$ are pure numbers measuring, respectively, pressure, vol-

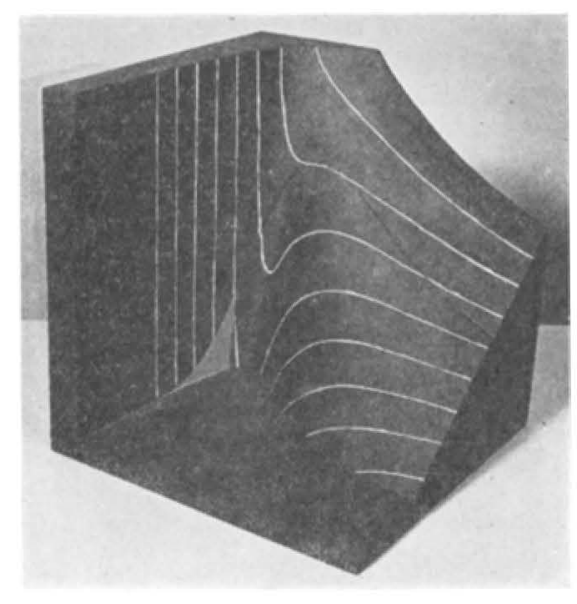

FIg. 2. The van der Waals surface.

ume and temperature referred to critical pressure, critical volume and critical temperature as unity. If the boards are about $\frac{1}{2}$ in. thick, a good result will be obtained by plotting an isotherm for every $1 / 20$ of critical temperature from $T=0$ to $T=24 / 20$. In Fig. 1, the isotherms represented with white (painted) lines on the black surface (finished with "blackboard" paint) are spaced at intervals of $1 / 10$ of critical temperature. When the boards have been sawed along the isothermal profiles, the pieces which are to form the model are coated with glue and nailed together in correct register. Any excess glue remaining in the steps between isotherms should be carefully removed. The remaining pieces of the boards are then stacked up without glue so as to nest loosely into the steps on the model, the whole forming a roughly cubical block. This is then clamped tightly between plane wood or metal sheets of sufficient stiffness to exert a uniform pressure all over and insure that the model will dry with the isothermal surfaces plane. It is a good idea to insert sheets of paper between the pieces of board which are later to be removed after the 


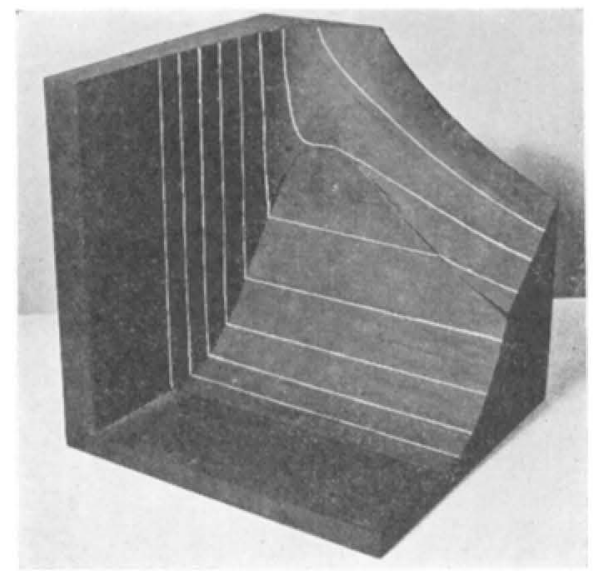

Fig. 3. Model with the second block in place.

glue has dried, to prevent any excess glue which has squeezed out under pressure from sticking to them. The stepped approximation to the van der Waals surface must now be dressed down with a wood file until the salient corners are entirely removed and the surface is just in contact with the plotted isotherms. Fortunately, the van der Waals surface is a "ruled surface"; all its profiles along lines of constant volume are straight lines. The wood file, therefore, should be held so that it strokes the model along these straight lines of constant volume. It is well to select a wood file with a "half round" cross section and to employ the round side. A "rattail" file of rather small diameter will be required for finishing the valley corresponding to the sharply pointed minima of the isotherms. In the model of Fig. 2, the plane representing zero volume is the vertical surface of the model at the back to the left, and the thickness of this vertical slab at the front edge (in the plane of zero temperature) represents the "covolume" which is one third of the critical volume. The slabs on which the isotherms were plotted were so dimensioned that the correct thickness to give this "covolume" could be obtained by gluing a flat sheet of wood on the face (in Fig. 2, invisible on the left-hand back side) so as to cover the end grains of the slabs and strengthen the model. As can readily be seen, the laminated structure of the model need only extend down to $T=0.3$, or 14 board thickness below critical temperature, the remainder of the left-hand wall being filled out with a flat piece of wood dressed down with the file to a continuation of the van der Waals surface. The model is eventually glued to a base board, shown in Fig. 2, whose upper face is the plane of zero pressure. Before this is done, however, a band-saw cut must be made to remove that portion of the model which fails to express the behavior of a substance when two phases coexist. This cut is faintly visible in Fig. 2. Unless the nails have been very judiciously placed in the laminated structure, it will be safer to make this cut with a metal band saw.

To determine the profile of this cut (projected on the $V, T$ plane), it is convenient to have the entire set of isotherms plotted on a sheet of paper to the same scale as that adopted for the model. Indeed, this plot should be made before plotting the isotherms on the wood, and they can then be traced on the wood slabs directly from the plot by means of carbon paper. It is well known that the region of each isotherm over which two phases (vapor and liquid) can coexist is a straight horizontal line of such height (pressure) that the loop of the van der Waals isotherm below the line includes the same area as the loop above the line. The pressure corresponding to the height of this line is the boiling pressure for that isotherm. These lines can very quickly be located in height on the paper plot by trial-and-error testing of the equality of the areas with a planimeter. The volumes at the two termini of each line are then read off for each temperature (isotherm) and this immediately gives the necessary data for plotting the profile of the cut projected on the $V, T$ plane. The cut surface both on the removable block and on the

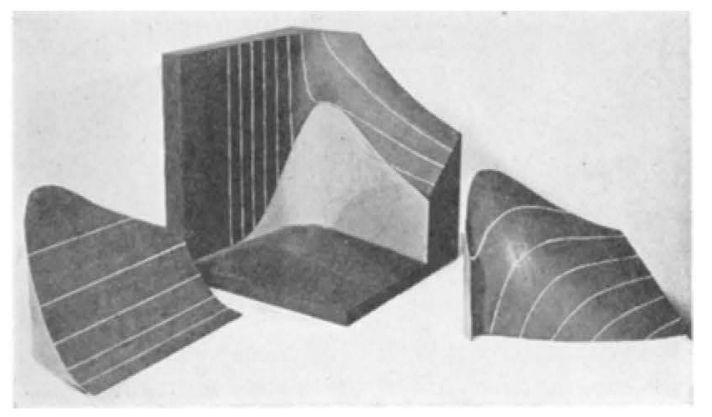

FIG. 4. Model with the two blocks removed. 
remainder of the model is painted red to distinguish it from the van der Waals surface. A second block is now prepared to fit against the cut surface of the model and this one is provided with an outward surface such that the isotherms in the two-phase region are horizontal. This is shown in place in Fig. 3 . When the model is shown to a class, it can be pointed out that the profile of this second block projected in the $P, T$ plane gives the curve of vapor pressure as a function of temperature, or the boiling curve. This affords a good opportunity also to illustrate the behavior of the pressure as the substance is heated at constant volume, the discontinuity in slope at the cut being very evident. The meanings of the critical point and of the critical isotherms become very clear when the model is viewed with this block in place, as does also the idea of the continuity of liquid and vapor states.

Figure 4 shows the model with the two blocks removed. The first-mentioned block representing the van der Waals equation has a portion on the left-hand side so thin that it is scarcely possible to represent it even in wood. As can be seen in Fig. 2, the absence of this portion leaves a small quasi-triangular area of the cut uncovered, a minor defect which is readily forgiven by the students.

\title{
Reproductions of Prints, Drawings and Paintings of Interest in the History of Physics
}

\author{
17. Frescos in the Tribuna di Galileo, Part I \\ E. C. Watson \\ California Institute of Technology, Pasadena, California
}

$T$ HE three frescos in the compartments of the domed ceiling of the innermost room of the Tribuna di Galileo ${ }^{1}$ depict three momentous periods in GaLILEo's life-the rising, the zenith and the setting of his genius. They are the work of Luigi Sabatelli.

In the first, reproduced in Plate 1, the young student is watching intently a swinging lamp in the Cathedral of Pisa (the inclined flame shows that the lamp is actually swinging). The face of the young Galileo is very expressive and the position of the hands-a device previously used by RAPHAEL-indicates that he is following a train of thought. So absorbed is he that his hat has fallen from his hand and is seen lying near his feet. According to the well-known story (probably apocryphal) GaLILEO timed the swings with the only watch he possessed, his own pulse, and thus discovered the isochronism of

${ }^{1}$ See Reproduction 16 of this series.

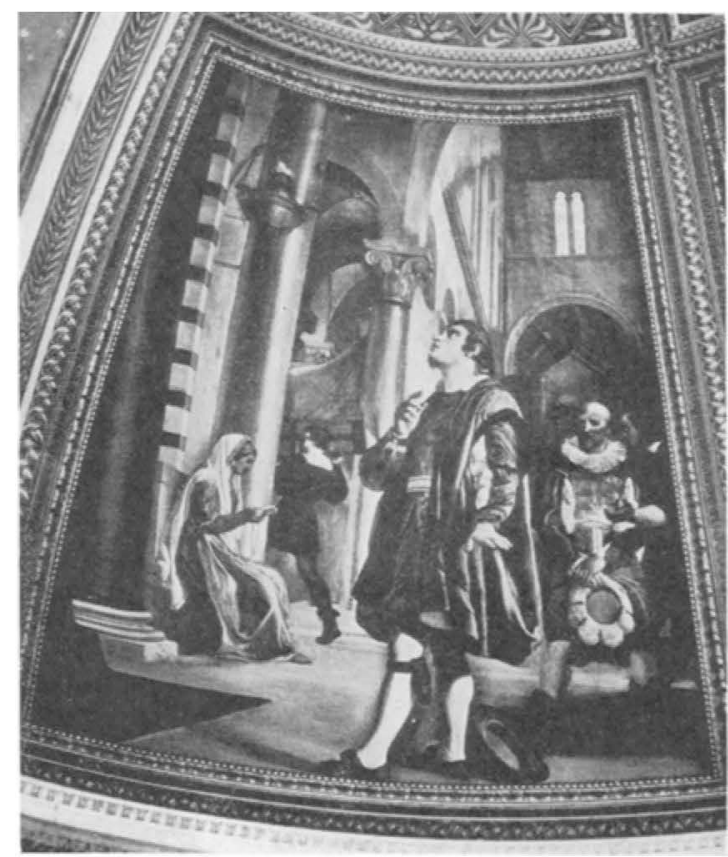

Plate 1. Galileo discovering the isochronism of the pendulum. [From the fresco in the Tribuna di Galileo.] 